\title{
Balance and Sound Conditions in Adults with Bilateral Cochlear Implants
}

\author{
Ross Tonini ${ }^{a}$ Helen S. Cohen ${ }^{a}$ Ajitkumar P. Mulavara ${ }^{b}$ \\ Haleh Sangi-Haghpeykar ${ }^{\mathrm{C}}$ \\ a Bobby R. Alford Department of Otolaryngology - Head and Neck Surgery, Baylor College \\ of Medicine, Houston, TX, USA; ${ }^{b} \mathrm{KBRwyle}$, Houston, TX, USA; ${ }^{\mathrm{c}}$ Department of Obstetrics \\ and Gynecology, Baylor College of Medicine, Houston, TX, USA
}

\begin{abstract}
What Is It about?
Some research suggests that sounds may affect standing balance. We tested the standing balance of 4 subjects who each had cochlear implants in both ears. They stood with eyes closed on compliant foam, so that the support surface was continuously unstable, sometimes when the person was moving his head, which is very challenging. Subjects heard no sound at all, minimal background noise, more background noise, and English and Romanian speakers. No sound and background noise did not affect balance skills. Balance decreased when people listened to either language. Therefore, being distracted by paying attention to words can affect balance.
\end{abstract}

\section{Keywords}

Postural control $\cdot$ Romberg $\cdot$ Dual task performance $\cdot$ Attention

\begin{abstract}
Purpose: To determine if (1) balance is impaired in patients with bilateral cochlear implants compared to healthy controls and (2) the presence of sound, non-speech, or speech affects standing balance. Materials and Methods: Four patients with bilateral cochlear implants were tested on three balance conditions on Romberg tests on medium-density compliant foam with eyes closed, with head stationary or moving in yaw or pitch, under 5 sound conditions: no sound, ambient background noise, pink noise, foreign language, English language. Results: Dependent measures were duration of standing and kinematics. Three of four subjects performed well with head still and no sound, background noise, or pink noise. All subjects performed poorly during the head movement conditions when hearing either foreignlanguage or English words. Subjects could not perform enough head movements during yaw and pitch conditions for accurate kinematic measurements. Conclusion: The no-sound condition did not influence standing balance skills. The addition of ambient or pink noise also did
\end{abstract}


not affect their balance. However, when subjects were distracted by paying attention to words, regardless whether or not they understood the words, standing balance skills deteriorated. Thus, distracted attention in these patients leads to impaired balance, which may impair functional motor skills.

(c) 2019 The Author(s)

Published by S. Karger AG, Basel

\section{Introduction}

The influence of hearing on balance is unclear. Some older studies have shown that moving auditory fields cause increased postural sway during quiet standing, especially in older adults $[1,2]$. The frequencies of the stimuli in those studies are unknown. More recent work showed that in elderly subjects hearing a rotating auditory sound that was meaningful, i.e., a story, was associated with reduced postural sway, but hearing a rotating non-meaningful sound, the musical note A at $440 \mathrm{~Hz}$, was not associated with increased sway [3]. Describing a study that used a $500 \mathrm{~Hz}$ auditory signal during posturography testing, the authors concluded that the response was probably vestibular rather than cochlear in nature [4]. The $440 \mathrm{~Hz}$ signal used by Deviterne et al. [3] may have stimulated the saccule as well as the cochlea, as in vestibular evoked myogenic potentials, although the speech sounds would have had a much wider range. Research on vestibular evoked myogenic potentials has shown that low-frequency sounds influence the utricle and saccule and generate minute but recordable postural responses in the sternocleidomastoid muscles [5]. A slight but ill-defined effect of some musical tones was found in a study of middle-aged normal subjects who heard particular musical tones imbedded in a musical composition [6]. By contrast, normal young adults in their 20s have been shown to have reduced postural sway when listening to a moving white noise stimulus compared to a stationary stimulus or ambient noise [7]. In general, these studies show a slight effect of auditory stimuli in normal adults. When normal young adults were tested on the Clinical Test of Sensory Integration on Balance (CTSIB) with pink noise $(100 \mathrm{~Hz}-4 \mathrm{kHz})$ in the background, subjects were more reliant on visual cues than when hearing was unavailable [8]. Furthermore, two different groups have shown that in normal subjects, postural sway was increased when ambient noise was unavailable $[9,10]$. Recent work has also shown that postural sway decreases in seniors and young adults in the presence of white noise versus ambient noise [11].

The cognitive load inherent in hearing sounds may have determined the auditory influence on balance. Audio biofeedback has been shown to reduce postural sway during quiet standing with eyes closed in patients with bilateral vestibular impairment [12]. The frequency of that audio biofeedback might have been the same $400 \mathrm{~Hz}$ signal used in a subsequent study with that patient population [13]. In other studies, the same authors showed that an oscillating sound, from 20 to $50 \mathrm{~dB}$ SPL ( 400 to $1,000 \mathrm{~Hz}$ ), was effective in reducing postural control during quiet standing in healthy subjects with intact vestibular systems [14], but a simpler signal at $400 \mathrm{~Hz}$ was also effective [15]. A different group also used an auditory signal, 60-95 dB SPL, to cue bilateral vestibular loss subjects about angular position or velocity compared to no feedback [16]. Subjects given the auditory feedback reduced their postural sway. Thus, vestibularly impaired subjects can learn to use an auditory biofeedback signal.

In subjects with known hearing impairments who wore hearing aids, the performance on quiet standing with eyes closed on foam, i.e., the modified Romberg or CTSIB $[17,18]$, was improved in the presence of broadband white noise $(0-4 \mathrm{kHz})$ compared to without sound [19]. A study of cochlear implant users showed that these subjects were impaired on stabilometry with head still or during head movement conditions compared to normal controls; 
whether or not they could hear ambient noise, or their implants were off so that they heard nothing [20]. Another study in bilateral or bimodal cochlear implant users showed that with their implants turned on in the presence of white noise $(0-4 \mathrm{kHz}, 65 \mathrm{~dB})$ postural sway was reduced compared to the test condition with implants turned off [21].

We tested subjects with bilateral cochlear implants on CTSIB using 5 different sound conditions and 3 different head movement conditions. The goal of the study was to determine if patients with bilateral cochlear implants have impaired standing balance compared to norms established with healthy controls tested previously [18] and to determine if the presence of sound, particular types of sound, or speech, affects standing balance.

\section{Materials and Methods}

\section{Subjects}

Subjects were patients who had bilateral cochlear implants, with the devices implanted at least 2 months apart. Subjects had no history of vertigo, central neurological lesions, peripheral neuropathy, or significant musculoskeletal problems. All subjects had been implanted with Cochlear Corporation implants (Cochlear Corporation, Sydney, NSW, Australia) and all were tested using the Cochlear Nucleus 5 speech processor with their routinely used map without modification for this study. No subjects had residual hearing after implantation for either ear. All subjects were ambulatory without gait aids. They were all fluent in English but did not speak Romanian. They gave written informed consent prior to participation. This study was approved by the Institutional Review Board for Human Subjects Research for the senior author's institution.

\section{Instrumentation}

All subjects were tested in an Otometrics (Quebec, QC, Canada) double-wall acoustically treated sound booth $(1.96 \mathrm{~m} \times 2.25 \mathrm{~m} \times 1.98 \mathrm{~m})$. They stood on a medium-density Sunmate compliant foam square $(0.46 \mathrm{~m} \times 0.46 \mathrm{~m}), \mathrm{PSI}=93$ (Dynamic Systems, Leicester, NC, USA). The back edge of the foam block was $1.52 \mathrm{~m}$ from the corner of the sound-treated room facing a sound field speaker at a height of $1 \mathrm{~m}$ that was $1.34 \mathrm{~m}$ from the person standing on the foam block.

\section{Test Conditions}

Subjects were tested under 5 sound conditions: (1) No sound (both processors off); (2) Ambient background noise only - bilateral processors on, (3) Speech-weighted noise with bilateral implants on, (4) Conversational speech - bilateral implants on (Romanian language sample), (5) Conversational speech - bilateral implants on (English language sample). The digitally recorded Romanian speech sample was a 2-min weather forecast in Romanian spoken by a male native Romanian speaker of the faculty of this department. Romanian was used because it is easily recognizable as a language, but our participants were unlikely to be familiar with it. The English language sample was a digitally recorded version of the Rainbow Passage [22] read by a male speaker (Otosuite Audiometry Module, GN Otometrics, 2011).

Prior to balance testing, the speech reception threshold of each subject was measured in the binaural configuration in the sound field. Subsequently, for all sound conditions, the stimulus sound was presented at an intensity level that was $30 \mathrm{~dB}$ greater than each subject's measured bilateral speech reception threshold in the sound field, so that the subjects could hear the stimuli. The noise stimulus in condition 3 and the speech stimuli from condition 4 and condition 5 were all presented at $0^{\circ}$ relative to the subject. 
Table 1. Subject details: age at test, internal device and external device per ear

\begin{tabular}{|c|c|c|c|c|}
\hline Subject & $\begin{array}{l}\text { Age, } \\
\text { years }\end{array}$ & Right ear & Left ear & $\begin{array}{l}\text { Test time from first } \\
\text { implant, months }\end{array}$ \\
\hline 1 & 48 & $\begin{array}{l}\text { Freedom CI24RE Contour } \\
\text { Advance (Nucleus } 5)^{1}\end{array}$ & $\begin{array}{l}2.25 \text { months }^{2} \\
\text { Freedom CI24RE Contour } \\
\text { Advance (Nucleus 5) }\end{array}$ & 54.5 \\
\hline 2 & 65 & $\begin{array}{l}21.25 \text { months }^{2} \\
\text { Freedom CI24 Contour } \\
\text { Advance (Nucleus 5) }\end{array}$ & CI512 (Nucleus 5) ${ }^{1}$ & 64.75 \\
\hline 3 & 70 & CI24RE (Nucleus 5) ${ }^{1}$ & $\begin{array}{l}1.75 \text { months }^{2} \\
\text { CI24RE (Nucleus 5) }\end{array}$ & 146.1 \\
\hline 4 & 79 & $\begin{array}{l}\text { CI24RE Nucleus } 24 \text { Contour } \\
\text { (Nucleus 5) }\end{array}$ & $\begin{array}{l}104.75 \text { months }^{2} \\
\text { Freedom CI24RE Contour } \\
\text { Advance (Nucleus 5) }\end{array}$ & 149.25 \\
\hline
\end{tabular}

${ }^{1}$ First implant. ${ }^{2}$ Second implant, length of time from first to second implants, length of time from first implant to test in this study.

Subjects wore socks but no shoes. They stood on the foam with feet next to each other and arms crossed. A $0.33 \mathrm{~Hz}$ tone was used to cue head movement trials using an auditory signal that oscillated between 170 and $450 \mathrm{~Hz}$ at a comfortable intensity level via the amplifier on a laptop computer. For no-sound trials, when subjects could not hear the auditory cue from the computer, to cue head movements, the subject held a smart phone running a $0.33 \mathrm{~Hz}$ signal on the Vibronome vibrating metronome app. Before the head movement trials, subjects practiced moving the head movements while standing on the floor and holding the smart phone or hearing the tone. When fatigued, they were allowed to sit down to rest.

We attempted to obtain kinematic data by having subjects wear two lightweight inertial motion units (Xsens North America, Inc., $5.25 \times 3.75 \times 2 \mathrm{~cm}$, weight $28.3 \mathrm{~g}$ ) centered on the head with a headband and centered on the mid-torso back with a lightweight vest, as in previous work [18]. We also counted the number of head movements during head movement trials by observation.

\section{Protocol}

All balance tests were given in the same order, with trials lasting up to $30 \mathrm{~s}$ : head still, head moving in yaw (shaking "No", left to right), nodding in pitch (nodding "Yes", up-down) per established protocol [18]. Sound conditions were given in pseudorandom order to avoid potential order effects. Dependent measures were the length of time the subject could perform the trial and the number of head movements the subject could make during pitch and yaw head movement trials.

\section{Statistical Methods}

At each head movement condition (still, yaw, pitch), we assessed the association between trial duration (study outcome) and various sound conditions (no sound, background noise, pink noise, a paragraph in Romanian, a paragraph in English). This was done by using linear mixed methods where we compared least square means of trial duration between different sound conditions. All analyses were performed in SAS (Version 9.4, Cary, NC, USA). 


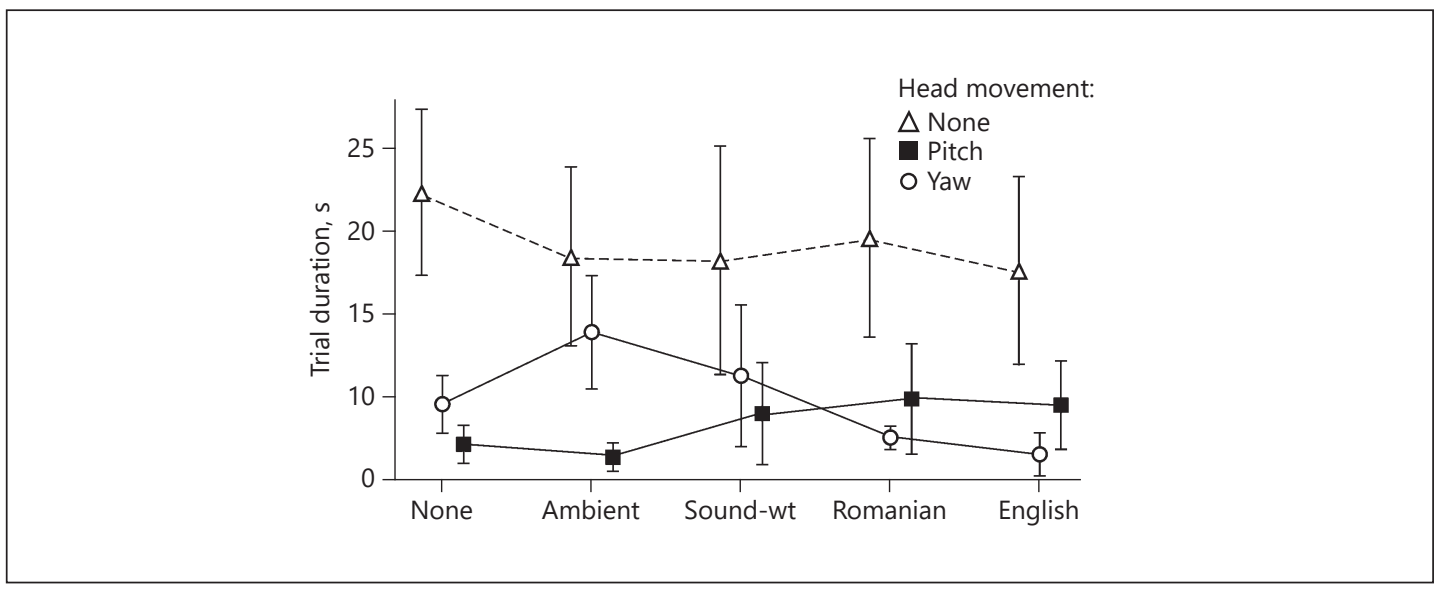

Fig. 1. Mean trial duration influenced by sound and head movement conditions. Error bars are standard errors. The symbols for the yaw and pitch head movement conditions have been displaced rightward from the symbol for head still for improved visualization of the symbols and error bars. Sound conditions were None (processors off), ambient background noise (Ambient), speech-weighted noise (Sound-wt), Romanian language passage (Romanian), and English language passage (English). Head movement conditions were head still (None), head moving at $0.33 \mathrm{~Hz}$ in yaw (Yaw), and head moving at $0.33 \mathrm{~Hz}$ in pitch (Pitch).

\section{Results}

The sample included 4 patients: 2 males and 2 females, aged 48, 65, 70, and 79 years. Although other patients seen in this clinic have had bilateral cochlear implants, no other patients met all of the inclusion criteria. Subjects' ages, relative dates of surgery and testing, and implant details are shown in Table 1. The treating neurotologists were unable to make definitive diagnoses, but all 4 subjects had documented, progressive sensorineural hearing loss; none had Ménière's disease.

At each sound condition, the longest trial duration was found when the head was still $(p<0.0001)$, as shown in Figure 1. With the exception of the 48-year-old subject, on the no-sound condition with the head still, subjects stood for longer than most healthy controls under that condition. The 48-year-old subject's performance was better in the ambient noise and pink noise conditions with the head still. As shown in Figure 1, at the head still or pitch condition, no differences in trial duration were seen across the various noise conditions. With the head moving in yaw, no differences were found between the background noise and pink noise conditions, but there was a trend for decreased trial durations when subjects heard Romanian (6.4 s difference, $p=0.1$ ) and English (7.4 s difference, $p=0.06$ ). No such changes were found with the head moving in pitch (see Fig. 1).

To calculate kinematic measures accurately, we needed 5 cycles of head movements. Similar to many hearing subjects with chronic vestibular impairments [18], these subjects were unable to generate enough head movements during yaw and pitch trials. No apparent differences were found during the head still trials. Therefore, the kinematic analyses will not be discussed further.

The number of head movements might have indicated the difficulty of the task. In the yaw rotation trials, subjects made significantly fewer head movements in the Romanian language condition compared to the background noise condition $(p=0.03)$, and in the English language condition compared to the background noise condition $(p=0.019)$. In the pitch rotation trials, no differences were found in any of the sound conditions (see Fig. 2). 


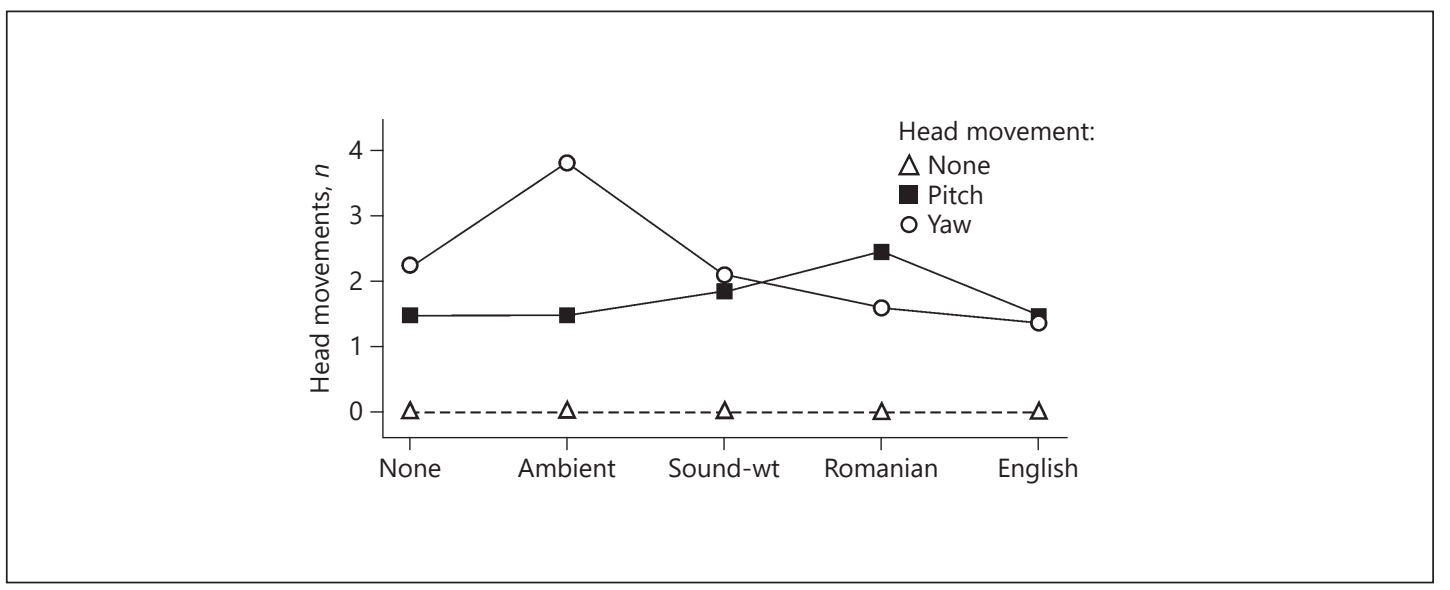

Fig. 2. Mean number of head movements influenced by sound and head movement conditions. Sound conditions were None (processors off), ambient background noise (Ambient), speech-weighted noise (Sound-wt), Romanian language passage (Romanian), and English language passage (English). Head movement conditions were head still (None), head moving at $0.33 \mathrm{~Hz}$ in yaw (Yaw), and head moving at $0.33 \mathrm{~Hz}$ in pitch (Pitch).

\section{Discussion/Conclusion}

Although no post-implant audiograms under insert earphones are available for any of the patients included in this series, based on a review of their pre-implant audiograms, and considering the era during which they were implanted and the electrodes with which they were implanted, we have no expectation that they would have had any meaningful lowfrequency detection in either of the implanted ears. Previous research has shown that the presence of a low-frequency sound has no effect on measures of balance in normal-hearing adults [23], but high-frequency sound does have an effect of reduced postural sway. Therefore, the presence or absence of known low-frequency hearing in our study group is not the reason for the effects observed in this study. The probable lack of low-frequency hearing in our subjects and the lack of differences on the no-sound, background noise, and pink noise conditions is consistent with the existing literature. Our data suggest that the absence of sound, per se, does not impair simple standing balance. Unlike previous work with normal subjects [8-11], or recent work with cochlear implant users [21], our small sample of bilateral cochlear implant users had no change in performance time across the sound conditions of absence of noise, ambient noise, or pink noise.

The lack of attention to sound may even have conferred a slight advantage in older people during quiet standing with the head still. Quiet standing, even without apparent dual tasking, is more challenging for older people than younger people $[18,24]$. Dual tasking is also more challenging for older people than younger people [25]. The three older subjects all had balance at least as good as comparable hearing healthy controls [18]. The presence of ambient noise during head-still conditions did not affect performance significantly or even noticeably. Thus, the absence of the need to attend to anything other than maintaining balance may have helped the older subjects. The cochlear implant, per se, would not have conferred an advantage, but the total absence of sound might have provided a less distracting experience, with a hitherto unknown type of dual-tasking paradigm. Perhaps hearing some routine background noise actually does provide a type of dual-tasking for older subjects, as older adults are known to perform dual-tasking during balance worse than younger adults [26]. That idea has not been previously tested. 
The effect of listening to speech was different. Listening to a voice provides a kind of cognitive task, so that listening to a voice and balancing is a type of dual tasking. In some elderly people, conversation during walking has been shown to be problematic [27, 28]. The English and Romanian language conditions presented such a dual-task problem, demanding divided attention. Similar to a previous study [3], we found differences in balance when subjects listened to language compared to their responses when they listened to noise. The results in the present study show that the language does not have to be intelligible to the listener. People tend to listen to words, probably trying to understand them, suggesting that sound with no meaning attached, i.e. the kind of random, ambient noise that is present constantly in our lives, has minimal influence on attention or balance during a balance task that is not very challenging.

The data in the present study support the idea that multitasking caused by adding a cognitive load makes that task more challenging. The cochlear implant, per se, does not cause a balance problem. Instead, because these people were so hearing-impaired, they had to work harder to derive meaning from sound when they knew the meaning should be there. In so doing, attentional resources were diverted from the novel and challenging balance task, thus making the balance task more difficult.

When subjects heard sentences spoken in either language, one in which they were fluent and one which they had never heard, in the yaw head movement trials, their balance tended to deteriorate. Thus, being distracted by speech while standing impairs simple balance skills in the yaw condition. These results, along with the finding that distraction did not affect balance during pitch head rotations, should be taken with caution as these interpretations are based on a small group of subjects. A study to investigate these issues systematically in a larger population is warranted. In tests of standing balance alone, pitch and yaw head movements appear to be equally challenging, but apparently the cognitive load is greater with yaw rather than pitch head movements.

The type of cognitive load may have affected performance under different sound conditions. Background noise poses a minimal cognitive load. Listening to language, whether comprehensible or not, poses a higher cognitive load. Fraizer and Mitra [26] reviewed the evidence about dual tasking and postural control and suggested that two possible mechanisms could be responsible for degraded performance during high cognitive load, either an absolute limit on cognitive capacity or a bottleneck in cognitive processing due to serial processing mechanisms. We are unable to determine which type of processing problem is most likely to be involved in this case.

Therefore, hearing-impaired patients should be careful when performing motor tasks in noisy environments if they will need to pay attention to some sounds such as voices or safety signals. These individuals might benefit from rehabilitation that introduces progressively greater cognitive sound challenges as an aspect of training dual-task performance during the execution of motor tasks, especially tasks that include balance components.

This study had some limitations. The sample size was small. In this institution few patients are implanted with bilateral cochlear implants. Of that limited group, fewer people met the inclusion criteria of being able to stand unassisted and without having joint replacements. Post hoc power analyses indicated that in the English language condition having 6 subjects would have yielded significant differences and in the Romanian language condition having 7 subjects would have yielded significant differences. Nonetheless, even with this small sample the magnitude of differences between the groups is large and the findings should be taken seriously.

Another minor limitation is that we used only one language which was unknown to subjects. Romanian is a Latin-based language and has some similarities to English. The results might have been different, perhaps even greater in magnitude, had we used a language that is more different from English, such as Chinese. 
Tonini et al.: Cochlear Implants, Balance, and Sound

\section{Acknowledgements}

We thank the staff of the Center for Hearing and Balance, Baylor College of Medicine, and Chris Miller, KBRwyle, for their assistance.

\section{Statement of Ethics}

Subjects gave written informed consent.

The study has been approved by the Institutional Review Board for Baylor College of Medicine and Affiliated Hospitals.

\section{Disclosure Statement}

The authors have no conflicts of interest to declare.

\section{Funding Sources}

This study was supported by NIH grant R01-DC009031 (HSC).

\section{Author Contributions}

Drs. Tonini and Cohen conceived the experiment. All authors contributed to the design of the experiment. Drs. Tonini and Cohen collected the data. Drs. Sangi-Haghpeykar and Mulavara analyzed the data. All authors contributed to interpreting the data. Dr. Cohen drafted the manuscript. Drs. Tonini, Mulavara, and Sangi-Haghpeykar contributed to revising the manuscript critically for important scientific content. All authors gave final approval of the submitted manuscript. All authors agree to be accountable for all aspect of the work in ensuring that questions related to the accuracy or integrity of any part of the work are appropriate.

\section{References}

1 Soames RW, Raper SA. The influence of moving auditory fields on postural sway behaviour in man. Eur J Appl Physiol Occup Physiol. 1992;65(3):241-5.

2 Tanaka T, Kojima S, Takeda H, Ino S, Ifukube T. The influence of moving auditory stimuli on standing balance in healthy young adults and the elderly. Ergonomics. 2001 Dec;44(15):1403-12.

3 Deviterne D, Gauchard GC, Jamet M, Vançon G, Perrin PP. Added cognitive load through rotary auditory stimulation can improve the quality of postural control in the elderly. Brain Res Bull. 2005 Jan;64(6):487-92.

4 Alessandrini M, Lanciani R, Bruno E, Napolitano B, Di Girolamo S. Posturography frequency analysis of soundevoked body sway in normal subjects. Eur Arch Otorhinolaryngol. 2006 Mar;263(3):248-52.

5 Fife TD, Colebatch JG, Kerber KA, Brantberg K, Strupp M, Lee H, et al. Practice guideline: Cervical and ocular vestibular evoked myogenic potential testing: Report of the Guideline Development, Dissemination, and Implementation Subcommittee of the American Academy of Neurology. Neurology. 2017 Nov;89(22): 2288-96.

6 Pagnacco G, Klotzek AS, Carrick FR, Wright CH, Oggero E. Effect of tone-based sound stimulation on balance performance of normal subjects: preliminary investigation. Biomed Sci Instrum. 2015;51:54-61.

7 Gandemer L, Parseihian G, Kronland-Martinet R, Bourdin C. The influence of horizontally rotating sound on standing balance. Exp Brain Res. 2014 Dec;232(12):3813-20.

8 Maheu M, Sharp A, Landry SP, Champoux F. Sensory reweighting after loss of auditory cues in healthy adults. Gait Posture. 2017 Mar;53:151-4. 
Tonini et al.: Cochlear Implants, Balance, and Sound

9 Kanegaonkar RG, Amin K, Clarke M. The contribution of hearing to normal balance. J Laryngol Otol. 2012 Oct; 126(10):984-8.

10 Vitkovic J, Le C, Lee SL, Clark RA. The contribution of hearing and hearing loss to balance control. Audiol Neurotol. 2016;21(4):195-202.

11 Ross JM, Will OJ, McGann Z, Balasubramaniam R. Auditory white noise reduces age-related fluctuations in balance. Neurosci Lett. 2016 Sep;630:216-21.

12 Dozza M, Chiari L, Horak FB. Audio-biofeedback improves balance in patients with bilateral vestibular loss. Arch Phys Med Rehabil. 2005 Jul;86(7):1401-3.

13 Dozza M, Horak FB, Chiari L. Auditory biofeedback substitutes for loss of sensory information in maintaining stance. Exp Brain Res. 2007 Mar;178(1):37-48.

14 Dozza M, Chiari L, Hlavacka F, Cappello A, Horak FB. Effects of linear versus sigmoid coding of visual or audio biofeedback for the control of upright stance. IEEE Trans Neural Syst Rehabil Eng. 2006 Dec;14(4):505-12.

15 Dozza M, Chiari L, Peterka RJ, Wall C, Horak FB. What is the most effective type of audio-biofeedback for postural motor learning? Gait Posture. 2011 Jul;34(3):313-9.

16 Hegeman J, Honegger F, Kupper M, Allum JH. The balance control of bilateral peripheral vestibular loss subjects and its improvement with auditory prosthetic feedback. J Vestib Res. 2005;15(2):109-17.

17 Shumway-Cook A, Horak FB. Assessing the influence of sensory interaction of balance. Suggestion from the field. Phys Ther. 1986 Oct;66(10):1548-50.

18 Cohen HS, Mulavara AP, Peters BT, Sangi-Haghpeykar H, Bloomberg JJ. Standing balance tests for screening people with vestibular impairments. Laryngoscope. 2014 Feb;124(2):545-50.

19 Rumalla K, Karim AM, Hullar TE. The effect of hearing aids on postural stability. Laryngoscope. 2015 Mar; 125(3):720-3

20 Bernard-Demanze L, Léonard J, Dumitrescu M, Meller R, Magnan J, Lacour M. Static and dynamic posture control in postlingual cochlear implanted patients: effects of dual-tasking, visual and auditory inputs suppression. Front Integr Nuerosci. 2014 Jan; 7:111.

21 Shayman CS, Mancini M, Weaver TS, King LA, Hullar TE. The contribution of cochlear implants to postural stability. Laryngoscope. 2018 Jul;128(7):1676-80.

22 Fairbanks G. Voice and articulation drillbook. New York: Harper and Brothers; 1937.

23 Siedlecka B, Sobera M, Sikora A, Drzewowska I. The influence of sounds on posture control. Acta Bioeng Biomech. 2015;17(3):96-102.

24 Cohen H, Heaton LG, Congdon SL, Jenkins HA. Changes in sensory organization test scores with age. Age Ageing. 1996 Jan;25(1):39-44.

25 Verhaeghen P, Cerella J. Aging, executive control, and attention: a review of meta-analyses. Neurosci Biobehav Rev. 2002 Nov;26(7):849-57.

26 Fraizer EV, Mitra S. Methodological and interpretive issues in posture-cognition dual-tasking in upright stance. Gait Posture. 2008 Feb;27(2):271-9.

27 Lundin-Olsson L, Nyberg L, Gustafson Y. "Stops walking when talking” as a predictor of falls in elderly people. Lancet. 1997 Mar;349(9052):617.

28 Hyndman D, Ashburn A. Stops walking when talking as a predictor of falls in people with stroke living in the community. J Neurol Neurosurg Psychiatry. 2004 Jul;75(7):994-7. 